\title{
Gold Nanoparticles on Polarizable Surfaces as Raman Scattering Antennas
}

\author{
Shiuan-Yeh Chen, ${ }^{t, \neq}$ Jack J. Mock, ${ }^{t, \neq}$ Ryan T. Hill, ${ }^{\S}$ Ashutosh Chilkoti, ${ }^{\S, \perp}$ David R. Smith, ${ }^{+, \neq}$and \\ Anne A. Lazarides ${ }^{ \pm, \varsigma, \|, *}$ \\ ${ }^{\dagger}$ Department of Electrical and Computer Engineering, ${ }^{\ddagger}$ Center for Metamaterials and Integrated Plasmonics, ${ }^{5}$ Center for Biologically Inspired Materials and Material \\ Systems, ${ }^{\perp}$ Department of Biomedical Engineering, and "Department of Mechanical Engineering and Materials Science, Duke University, Durham, North Carolina 27708, \\ United States
}

$\mathrm{R}$ aman scattering from molecules positioned close to metal surfaces is well-known to be enhanced when the substrates support surface plasmons and molecules are within the near fields of surface excitations. ${ }^{1-5}$ The surface plasmon modes that provide the field enhancement may also facilitate outcoupling of Raman emission. ${ }^{6,7}$ The high specificity of Raman spectra and their sensitivity to the local environment have motivated extensive investigation of the Raman scattering enhancing properties of structures that support surface plasmons..$^{8-17}$ Desirable properties of substrates for surface enhancement of Raman scattering (SERS) include geometries that support large polarization charge, provide robust control of resonances, and allow resonances to be tuned. Among plasmonic substrates that provide high field enhancement are structures with junctions such as pairs of particles or clusters ${ }^{1-5,8,18,19}$ and particles on polarizable films. ${ }^{20-29}$ Whereas structures designed to enhance fluorescence must balance field enhancement against quenching, ${ }^{30}$ SERS signals will be brightest when molecules are closer to substrates or in extremely narrow junctions that support intense gap fields. However, in these systems, SERS is strongly sensitive to junction width. ${ }^{18,19,24}$ Furthermore, quantitative excitation and enhancement requires not only control of junction width but usually also alignment of the SERS substrate relative to the polarization direction of the incident field. We previously have reported quantitative SERS at the ensemble level from solution phase assemblies that used molecular tethers to control junction width. ${ }^{31}$ In this bulk phase system, the problem of aligning structures with the incident field direction was circumvented through

www.acsnano.org

ABSTRACT Surface plasmons supported by metal nanoparticles are perturbed by coupling to a surface that is polarizable. Coupling results in enhancement of near fields and may increase the scattering efficiency of radiative modes. In this study, we investigate the Rayleigh and Raman scattering properties of gold nanoparticles functionalized with cyanine deposited on silicon and quartz wafers and on gold thin films. Dark-field scattering images display red shifting of the gold nanoparticle plasmon resonance and doughnut-shaped scattering patterns when particles are deposited on silicon or on a gold film. The imaged radiation patterns and individual particle spectra reveal that the polarizable substrates control both the orientation and brightness of the radiative modes. Comparison with simulation indicates that, in a particle - surface system with a fixed junction width, plasmon band shifts are controlled quantitatively by the permittivity of the wafer or the film. Surface-enhanced resonance Raman scattering (SERRS) spectra and images are collected from cyanine on particles on gold films. SERRS images of the particles on gold films are doughnut-shaped as are their Rayleigh images, indicating that the SERRS is controlled by the polarization of plasmons in the antenna nanostructures. Near-field enhancement and radiative efficiency of the antenna are sufficient to enable Raman scattering cyanines to function as gap field probes. Through collective interpretation of individual particle Rayleigh spectra and spectral simulations, the geometric basis for small observed variations in the wavelength and intensity of plasmon resonant scattering from individual antenna on the three surfaces is explained.

KEYWORDS: surface plasmon · surface-enhanced Raman scattering · individual nanoparticles $\cdot$ dark-field scattering $\cdot$ nanoantennas $\cdot$ super-radiance

use of symmetric satellite structures that incorporate interparticle junctions oriented in all directions. However, it would be desirable to control both junction width and orientation in structures on surfaces where the properties of single structures can be observed.

Recently, some of us have demonstrated control of plasmon resonance and polarization in a format that consists of a metal particle on a dielectric spacer layer on a metal film. ${ }^{32}$ This format provides intrinsic control of the orientation of the observable dipolar excitation, as evident from the doughnut shape of the particle image. The reproducible dependence of the plasmon resonance frequency of single nanoparticles on the thickness of the spacer layer points to good

\begin{abstract}
*Address correspondence to anne.lazarides@duke.edu.

Received for review July 14, 2010 and accepted October 25, 2010.
\end{abstract}

Published online November 1, 2010. $10.1021 / \mathrm{nn} 101644$ s

() 2010 American Chemical Society 


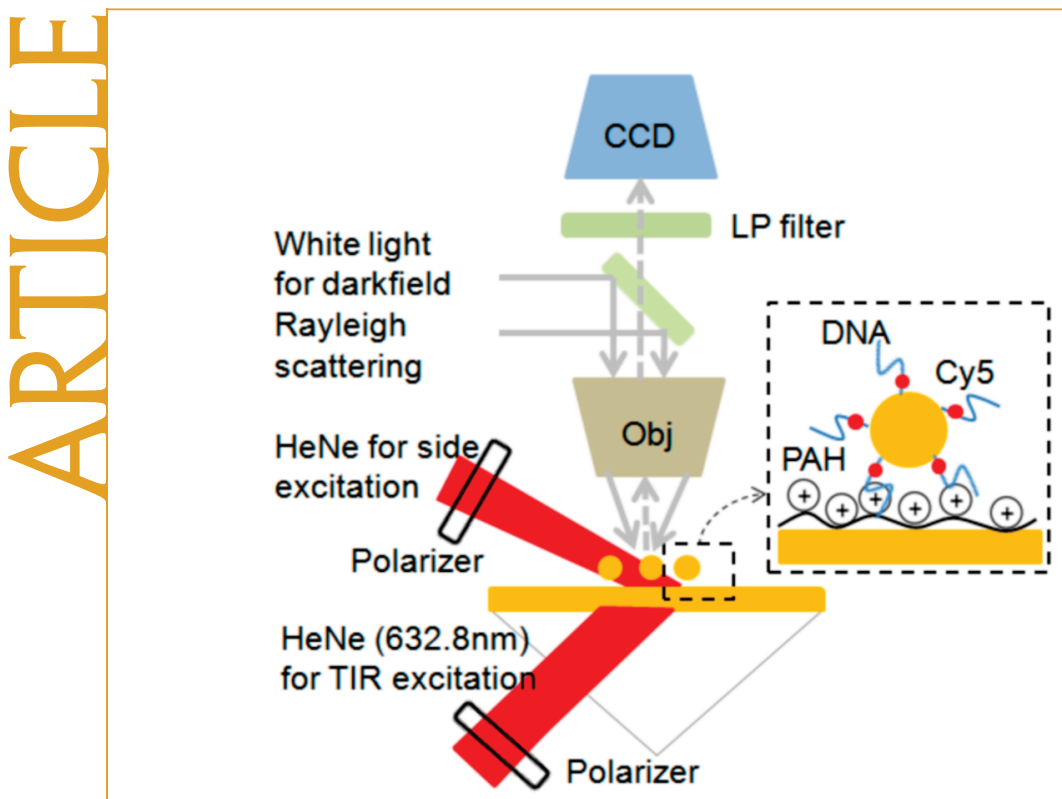

Figure 1. Illustration of the optical setup for collecting Rayleigh or Raman signals. White light excitation through the objective and two laser excitation schemes and the image collection path are shown. For collection of spectra, light is directed through a pinhole to a spectrometer (not shown). Inset: antenna system composed of a gold nanoparticle electrostatically coupled to a gold film. The Raman scatterers, Cy5, are tethered to the nanoparticle. The antenna gap width is controlled by a polyelectrolyte film.

control not only of the plasmon resonance but also of the separation between the particle and the film. As junction width is the variable to which gap fields are most sensitive, control of junction width implies control of field enhancement and suggests that the particle-film system is promising for quantitative SERS. Here we investigate both the directionality of scattering and the influence of surface composition on the scattering efficiency of particles on films.

Prior studies have documented control of plasmon resonance in particle on surface systems using thin films composed of lithium fluoride, ${ }^{33}$ silica, ${ }^{34-36}$ polymers, ${ }^{21}$ and self-assembled monolayers. ${ }^{24}$ These and other studies document red shifting of nanoparticle plasmons when metal nanoparticles are deposited on dielectric or metal surfaces. . $32,34-41$ The polarization of scattering from nanoparticle-surface systems has been characterized both by angle scanned detection ${ }^{34,35}$ and, more recently, by imaging. ${ }^{32,36}$ The role of discrete nanostructures in controlling the directionality of scattering has been studied implicitly through investigation of scattering from coupled structures under polarized illumination..$^{42-46}$ Ongoing research is devoted to understanding how these optical antenna systems control the spatial properties of emission. Several groups have studied how single metal nanostructures modify emission from single fluorescent molecules. Modification of both the rate and polarization of single molecule emission has been reported as a function of the molecule's relative position and proximity to a metal nanodisk. ${ }^{47}$ Redirection of emission from a molecule with a horizontally oriented transition dipole has been demonstrated using a vertical nanorod. ${ }^{48}$ The emissive properties of quantum dots and molecules positioned in junctions between nanoparticles also have been investigated on the single nanostructure level., ${ }^{4,18,19,49-52}$ These investigations of how antenna systems control molecular emission build upon prior studies of twophoton photoluminescence (TPPL) ${ }^{46,53-55}$ and inelastic scattering. ${ }^{38}$ Studies of both discrete coupled structures and particles on films provide compelling evidence of the effectiveness with which plasmonic nanosystems direct emission.

Here we adopt the cyanine-functionalized particles used previously as templates for assembly of bulk phase satellite structures ${ }^{31}$ for use in the planar format of a particle on a film. Under resonant illumination, cyanine in the near field of the most field enhancing coupled system displays surface-enhanced resonance Raman scattering (SERRS). We investigate the ability of metal, semiconducting, and dielectric surfaces to control frequency, intensity, and alignment of the brightest radiative plasmon mode and the role of the plasmon in controlling the spatial properties of SERRS. The antenna are gold nanoparticles positioned on one of two highly polarizable surfaces, gold and silicon; particles on quartz serve as a control.

\section{RESULTS}

Gold nanoparticles were functionalized with cyanine- and thiol-modified oligonucleotides as described previously. ${ }^{31}$ Fluorescence of the initial solutions and supernatants was monitored to quantify final coverage of cyanine (Cy5) on the particles. From the molecules adsorbed per particle, the area density of Cy5 molecules is one per $4 \mathrm{~nm}^{2}$. Gold films were deposited on quartz by e-beam evaporation. Polyelectrolyte, poly(allylamine) hydrochloride (PAH), was deposited on silicon and quartz wafers and on gold films. Prior to deposition of the cyanine-functionalized particles, the films and spacer layers were characterized using ellipsometry. The thickness of the native oxide layer on silicon was $1.83 \mathrm{~nm}$. In the region of the antenna resonance, relative to bulk values, ${ }^{56}$ the dielectric constant of the gold film displayed a larger imaginary part. Thicknesses of $0.56,0.13$, and $2.9 \mathrm{~nm}$ were found for the $\mathrm{PAH}$ on gold, silicon, and quartz. Thin PAH layers have been observed previously ${ }^{57}$ for $\mathrm{PAH}$ on silicon titanium oxide. However, uncertainties in the thickness estimates for PAH on silicon and on quartz are large due to low contrast between the PAH and either glassy or crystalline $\mathrm{SiO}_{2}$.

Dark-field color images were collected from nanoparticles deposited on the polyelectrolyte spacer/adhesion layer on all three surfaces, using a customized $\mathrm{Ni}$ kon dark-field (DF) microscope (Figure 1). A description of the setup is in the Methods section. Nanoparticles were illuminated by collimated white light passed 

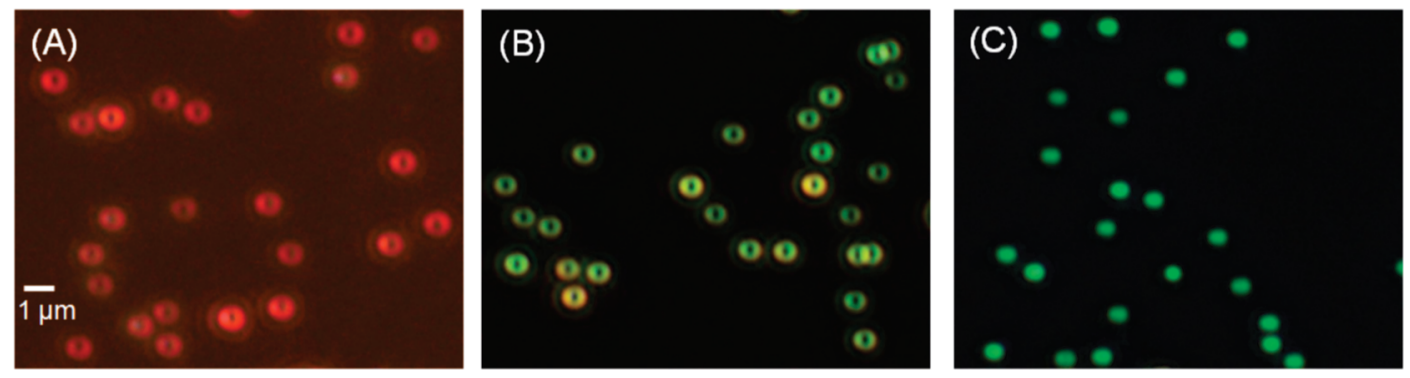

Figure 2. Dark-field images of gold nanoparticles on (A) a gold thin film, (B) a silicon wafer, and (C) a quartz wafer. Plasmon band position is displayed in the color of the images. The doughnut shape of the images of particles on gold and silicon indicates that, on high permittivity surfaces, scattering is dominated by a vertically polarized mode.

through and collected by the same objective (100X $\mathrm{DF}$, numerical aperture (NA), 0.9). Exposure times were $1.6 \mathrm{~s}$ for nanoparticles on gold and silicon and $5 \mathrm{~s}$ for nanoparticles on quartz because of the dependence of the cross section upon the polarizability of the substrate. Similar coverages of particles were observed on all substrates. Scattered light was red, yellow-green, and green from particles on gold, silicon, and quartz, respectively (Figure 2). Red shifting of the plasmon band is a signature of longitudinal particle-surface coupling. The colors, thus, provide a qualitative measure of the strength of interaction between nanoparticle plasmons and plasmons or more localized excitations at the surfaces, respectively, of gold, silicon, and quartz.

Gold nanoparticles on gold films and on silicon appear in dark-field Rayleigh images as doughnut shapes. The doughnut shape of the image indicates that radiation from each nanoparticle is associated with an oscillation oriented normal to the wafer or the film. ${ }^{58} \mathrm{Cou}-$ pling of the nanoparticles to a surface with high permittivity thus creates a population of nanoantennas whose orientations are well-defined. As the exciting field has components both perpendicular and parallel to the film, the doughnut-shaped profile and darkness of the doughnut center indicates enhanced scattering from vertical relative to horizontal modes. Enhancement of emission from a vertical electric dipole (VED) relative to a horizontal electric dipole (HED) previously has been observed both from molecules ${ }^{47,59}$ and metal nanoparticles close to a metal surface. ${ }^{32}$ Whereas the antenna resonance of nanoparticles on silicon is less red-shifted than the resonance of nanoparticles on gold, the polarizability of silicon is sufficient to produce an image with a doughnut-shaped point spread function. Images of nanoparticles on quartz do not display a spatial distribution characteristic of a VED, consistent with limited interaction with the surface, as indicated by their color, green.

To characterize more completely the properties of the nanoantennas, dark-field Rayleigh spectra were collected from the individual particles on all three surfaces and compared with simulation. Dark-field spectra were acquired using the same optical setup as was used for imaging except that output was directed through a different output port and dispersed by a spectrometer (Acton Spectrapro 2300i) onto a cooled CCD detector (Photometrics CoolSnap HQ). To allow collection of single particle spectra, a $200 \mu \mathrm{m}$ pinhole aperture is used. The integration time is $15 \mathrm{~s}$ for all spectra; thus, intensities reflect the relative scattering intensities of single particles on the three surfaces. The scattering signal from a single nanoparticle is corrected by the background collected from a nearby region without particles. Spectra from a single particle with a scattering intensity closest to the mean intensity from particles on each surface are shown in Figure 3a. Scattering band positions are 530,561, and $668 \mathrm{~nm}$ for particles on quartz, silicon, and gold, respectively, consistent with the colors of the Rayleigh images. Relative scattering intensities are approximately 1:5:25 for particles on the three surfaces, thus brightness is correlated with red shifting of the dominant mode. Previous studies of gold particles on dielectric surfaces have correlated the magnitude of the red shift of the resonance with the permittivity of the surface by invoking the image dipole approximation. ${ }^{39}$ Thus the longer wavelength of the resonance of particles on silicon (dielectric constant, $\varepsilon$ $\sim 16.3$ at resonance, $561 \mathrm{~nm}$ ) relative to that of particles
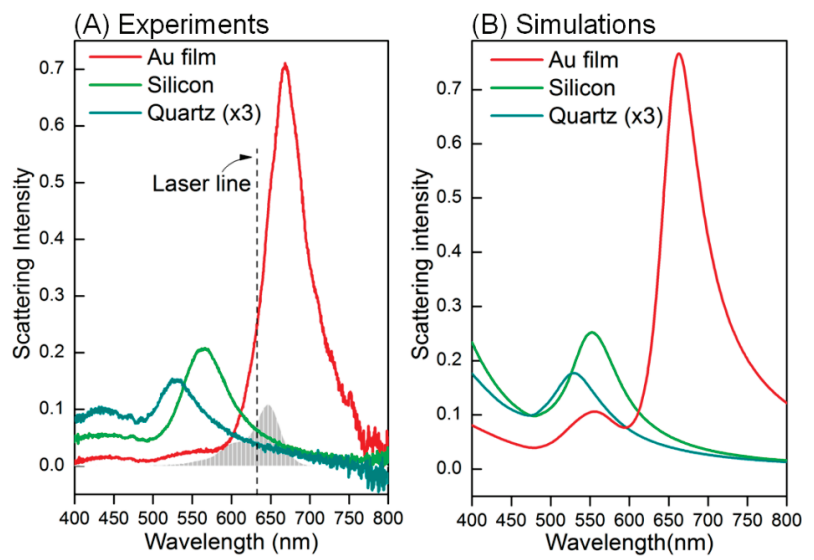

Figure 3. Single particle scattering spectra and spectral simulations of gold nanoparticles on a polyelectrolyte layer on quartz and silicon wafers and on a gold thin film. (A) Single particle spectra. Illumination and collection are through a $100 \times$ objective lens. Also shown is the absorption spectrum of the Cy5-modified oligonucleotide (shaded) and the laser line used for SERRS. (B) Simulated scattering spectra of a gold nanoparticle on each surface. Thicknesses of the dielectric spacer layers and the dielectric function of the gold film are as derived from ellipsometry. 


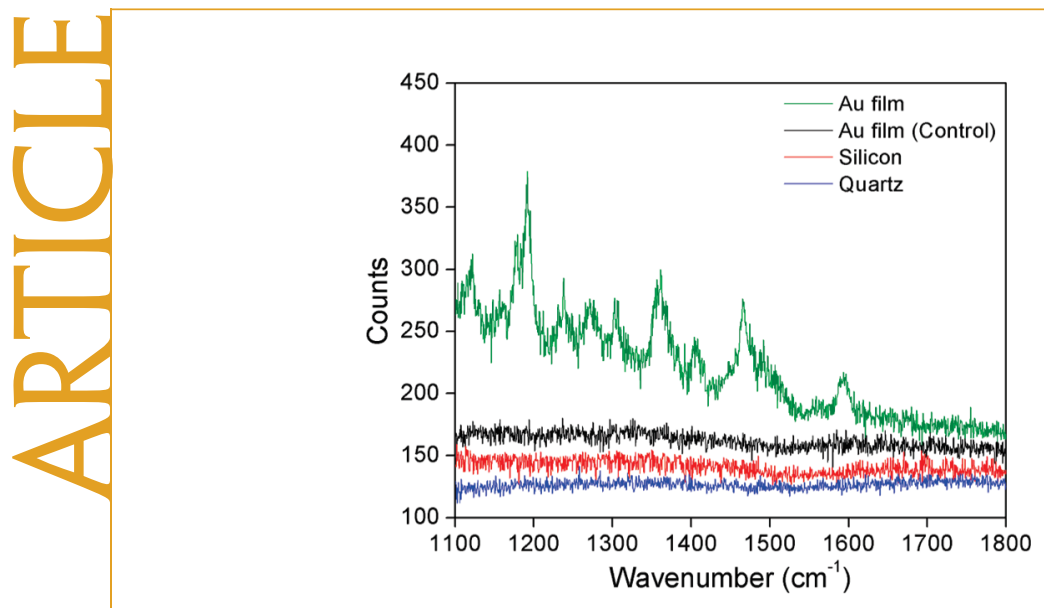

Figure 4. Raman spectra $\left(\lambda_{0}=632.8 \mathrm{~nm}\right)$ of Cy5functionalized gold nanoparticles on three surfaces: silicon, quartz, and a gold thin film. Raman features are detected from the particles on gold only. Scattering from control particles lacking Cy 5 on a gold film is also shown. The spectrum of the nanoparticles on the Au film is offset by 30 counts.

on quartz $(\varepsilon \sim 2.13)$ is as expected. However, the further redness of the primary scattering resonance of particles on a gold film relative to particles on silicon indicates that the radiative mode of a nanoparticle antenna is more strongly coupled to the gold film ( $\varepsilon_{668 \mathrm{~nm}} \sim$ $-14.1+1.02 i)$ than to silicon. The plasmon band of the gold on gold antenna displays some distortion, most likely due to Cy5 absorption. ${ }^{60-62}$

Inelastic scattering spectra in the Stokes range were collected from particles on all three surfaces. Inelastic scattering spectra were collected also from control nanoparticles on the gold film, using gold particles functionalized with oligonucleotides lacking the Cy5. For these measurements, a commercial Raman microspectrometer was used. A $632.8 \mathrm{~nm}$ laser light (8 $\mathrm{mW}$ ) on resonance with the Cy5 molecule was used to illuminate nanoparticles on all three surfaces. The laser

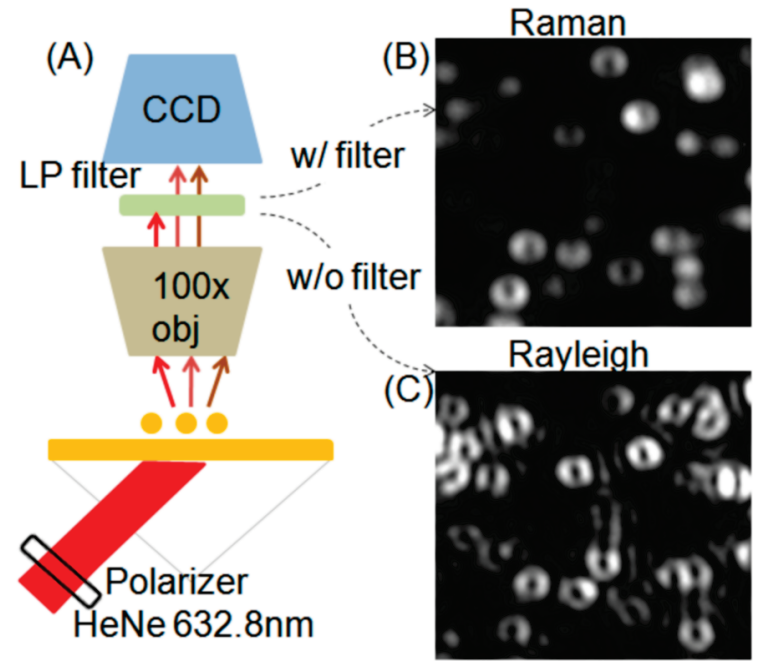

Figure 5. Raman and Rayleigh light scattering images. (A) IIlustration of the optical setup for acquiring single particle Raman and Rayleigh images, and (B) Raman and (C) Rayleigh images of single particles on gold films. The field of view is 5.6 $\mu \mathrm{m} \times 5.1 \mu \mathrm{m}$. line falls within the absorption band of Cy5 and within the primary scattering band of the gold nanoparticles on the gold film. Representative spectra are shown in Figure 4. Under red illumination, a Raman spectrum was collected from the substrate with the red surface plasmon, the substrate composed of gold particles on a gold film. The spectrum matches a bulk SERRS spectrum collected previously from nanoparticle cluster structures formed of the same Cy5-modified oligonucleotide nanoparticle conjugates, ${ }^{31}$ with no polyelectrolytes (PE) present. At this red excitation wavelength, Raman scattering from yellow-green or green particles on silicon and quartz substrates was not observed. Spectra collected from control particles with oligonucleotides lacking the Cy5 did not display detectable Raman peaks when deposited on another region of the PE on gold film. Thus neither DNA nor PE contributes to the Raman scattering observed.

Rayleigh and Raman laser light scattering images were collected from nanoparticles deposited on the polyelectrolyte spacer/adhesion layer on a gold film on quartz. A total internal reflection (TIR) excitation configuration was used owing to the small working distance of the $100 \times$ objective lens. Laser light $(30 \mathrm{~mW})$ at the same frequency used to collect Raman spectra was directed through the prism and sample substrate at $45^{\circ}$ and focused to a $100 \mu \mathrm{m}$ spot. Raman images (integration time, $20 \mathrm{~s}$ ) were collected using a long pass (LP) filter to remove the Rayleigh scattered light. Dark-field Rayleigh images were taken using a shorter integration time (1 s) under attenuated illumination to prevent saturation of the CCD. In the laser scattering image (Figure 5), as in the color image (Figure 2), gold nanoparticles on gold films have profiles with doughnut shapes. Under TIR illumination, as with excitation through the objective lens, the exciting field has components both perpendicular and parallel to the film. ${ }^{63}$ Nonetheless, under monochromatic as well as broadband illumination, the scattering pattern has the signature of a VED.

In the LP-filtered image, scattering is observed from the same locations as the sites from which Rayleigh scattering is observed. The Raman images, also, display a doughnut shape. This matching of spatial properties indicates that Raman scattering is governed by the radiative properties of the particle-surface plasmon. On a substrate that tightly localizes field intensity within a particle-surface gap, the molecules contributing to the Raman scattering will be the small number of molecules in the region of each gap with the highest electric field. Blinking of the Raman signals from many particles was observed (Figure 6). Blinking may signify motion of the particle or molecule or signify intermittency in Raman scattering from a single Cy5 molecule. As the duration of an on-state is comparable to the period required to collect a Raman signal, not all blinking antennas displayed blinking as clearly in a time trace as 
in the real time image or in a video (Supporting Information 1). A control experiment was performed using particles functionalized identically with a thiolated oligonucleotide that lacked the cyanine. Under the same excitation and collection conditions, surface-enhanced Raman scattering (SERS) signals were not observed (Supporting Information 2).

The doughnut-shaped point spread function of the Raman images of the SERS-active substrate, composed of gold nanoparticles on a gold film, demonstrates that, in this nanoantenna system, Raman emission has a scattering profile similar to the profile of a VED. To clarify the role of the vertical mode for Raman excitation, inelastic scattering spectra were collected in the custom microscope with the LP filter using s- and p-polarized and unpolarized (30 $\mathrm{mW}$ ) laser light. To control the incident light polarization more completely than possible using evanescent excitation, the laser beam is directed at the sample from the side and slightly focused to a 7.8 $\times 10^{3} \mu \mathrm{m}^{2}$ spot. Unpolarized incident power density thus was $380 \mathrm{~W} / \mathrm{cm}^{2}$. To accommodate the laser beam, a $50 \times(0.55 \mathrm{NA})$ objective with a longer working distance is used. Using the lower NA objective, scattering from a vertically oriented antenna is less efficiently collected; however, under unpolarized and p-polarized illumination, both Raman spectra and Raman images were observed (Figure 7). The p-polarization results in slightly lower signals than unpolarized excitation. However, all of the brighter Raman-active particles displayed Raman scattering under p-polarized excitation. The s-polarized excitation did not produce any observable SERRS. In this configuration, SERRS was collected also from smaller groups of scatterers under unpolarized illumination. Integrated intensity of the band at $1191 \mathrm{~cm}^{-1}$ collected by the $50 \times$ (NA 0.55) objective from three particles was $37.8 \mathrm{cps}$. Correction for the efficiencies of the optical components and the CCD yielded a corrected photon count of 750 photons per second (Supporting Information 7).

\section{DISCUSSION}

Overall, the experimental data confirm that surface properties are of key importance in determination of the band positions of the radiative resonances of metal particles on planar surfaces, and that the primary radiative mode of the gold particles is vertically polarized when the particle is positioned 1 or $2 \mathrm{~nm}$ from a gold or silicon. In addition, we observe that, for Raman scattering as for fluorescence, the directionality of scattering from molecules in surface-enhanced fields is controlled by the scattering properties of the radiative antenna surface plasmon modes. Prior fundamental studies of the coupling of particle and surface modes indicate that, at close spacings, the particle-surface system may support multiple coupled modes. ${ }^{64,65}$ However, not all modes are radiative, and band overlap may preclude detection of closely spaced modes. Rayleigh
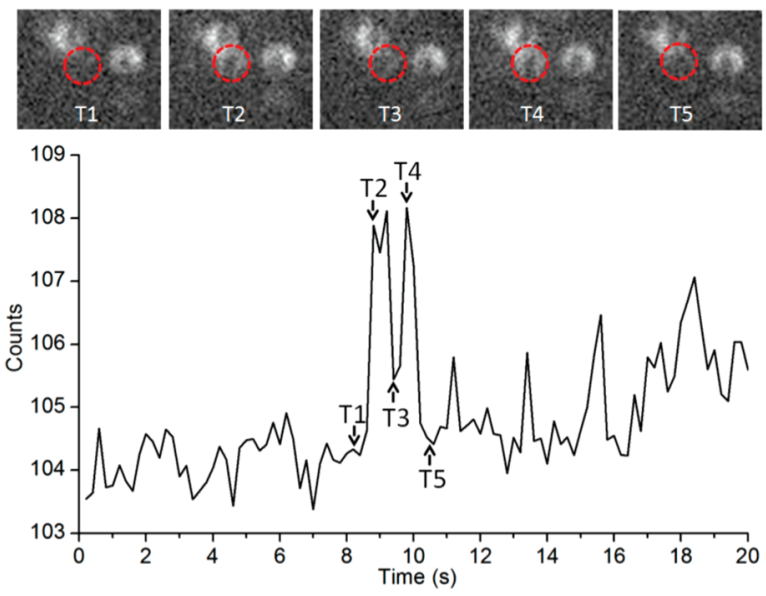

Figure 6. Intermittent Raman signal from a single nanoantenna. Time series of inelastic scattering from the Stokes shifted frequency range and series of images spanning several blinks.

spectra collected from the gold nanoparticle on gold film (Figure 3A) do reveal, however, not only a primary mode more than an order of magnitude brighter than the dipole mode of a nearly free particle on quartz but also a much weaker, higher energy mode at $\sim 560 \mathrm{~nm}$.

While the polarization of the brightest modes can be determined directly from the images, the character of the less bright modes may be inferred from their excitation by polarized light. Here we address the character of the radiative modes using simulation.

Particle-surface coupling was calculated and scattering spectra were simulated using the electrodynamic method of Bobbert and Vlieger. ${ }^{66}$ In this method, the vector spherical harmonic (VSH) modes of a sphere are coupled through the sphere's response to reflection by the surface of the VSH scattered waves (Supporting Information 3). Surface plasmon modes of like orientation

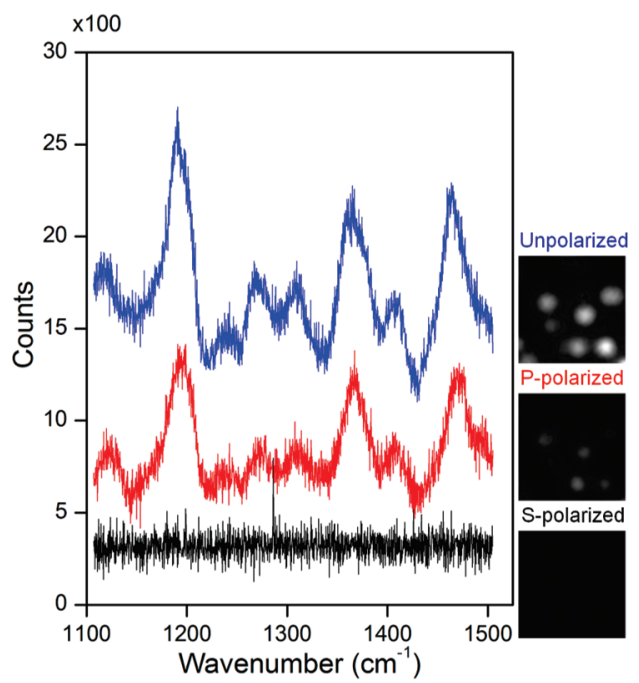

Figure 7. SERRS spectra and images of the nanoparticle on gold film under different excitation states: unpolarized (blue), p-polarized (red), and s-polarized (black). Spectra and images were collected through a $50 \times$ objective. The dimensions of the displayed images are $5.7 \mu \mathrm{m} \times 5.7 \mu \mathrm{m}$. Exposure times were $120 \mathrm{~s}$ for Raman spectra and $25 \mathrm{~s}$ for Raman images.

VOL. $4 \cdot$ NO. $11 \cdot 6535-6546 \cdot 2010$ 

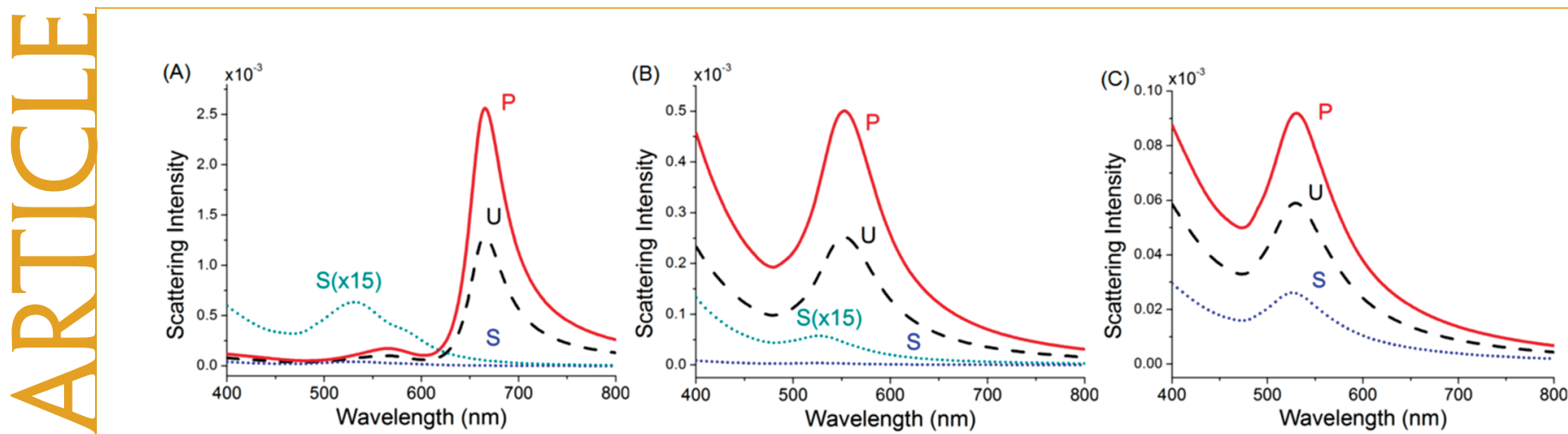

Figure 8. Simulated spectra of nanoparticles on three surfaces: (A) gold, (B) silicon, and (C) quartz under excitation with unpolarized (dashed line), p-polarized (solid line), and s-polarized light (dotted line). Angle of incidence is $70^{\circ}$ from normal; collection is of the light cone within $\sin ^{-1}(\mathrm{NA}), \mathrm{NA}=0.9$.

thus are mixed. In the model, particle size was 50.8 $\mathrm{nm}$. PAH and oxide layer thicknesses were as determined from ellipsometry. Initially, we reproduce spectra collected under unpolarized excitation. To simulate excitation from the dark-field objective lens, an incident wave $70^{\circ}$ from normal is used. To simulate collection through the $100 \times$ objective, the angular range of scattering included in the calculation is matched to the acceptance cone of the lens. Simulated scattering spectra for gold particles on the three surfaces are shown in Figure 3B. Plasmon band positions, widths, and relative intensities are reproduced extremely well. For all particle-surface systems, the average difference in band position between simulation and experiment is no more than several nanometers. Some cancellation of error may underlie this match, as the thickness and refractive index of the desiccated oligonucleotide particle-capping layer were not known and the dispersive property of the cyanine was neglected. However, the match of the scattering simulation to the observed spectra for particles on surfaces of all types confirms that, for a given antenna geometry, the complex permittivity of the surface controls both the intensity and wavelength of the primary scattering band. To clarify the effect on spectral features of the wider gap of the antenna on silicon compared with the antenna on the gold film, a spectrum was simulated also for a nanoparticle positioned as close to silicon as the nanoparticles are to the gold films (Supporting Information 4). The gap width variation accounted for only $\sim 10 \%$ of the difference in band position. A spectrum was calculated also for a nanoparticle on a substrate with the permittivity, $\varepsilon_{0}$, of free space using the same excitation/collection geometry. The calculation revealed that even a low permittivity substrate such as quartz reduces scattering under s-polarized illumination below that when the incident light is p-polarized. Relative to a free particle, scattering from the nanoparticle on a gold film is 2 orders of magnitude more intense. For the gold on gold film system, furthermore, the simulated spectrum includes the low intensity, shorter wavelength $(\sim 560$ $\mathrm{nm}$ ) band detected in the experimental spectrum (Figure $3 \mathrm{~A})$.
While the widths of resonances of nanoantenna composed of gold are too large to allow many bands to be resolved, the simulation capability provides an opportunity to investigate the substrate dependence of scattering from overlapping or low dipole moment modes. The response of the particle-film system under s- and p-polarization was simulated, although in the experiments, only unpolarized light was used. On quartz (Figure $8 \mathrm{C}$ ), the horizontal and vertical dipole modes are minimally split; however, compared with a nanoparticle on an $\varepsilon_{0}$ substrate, only the p-component is enhanced. On silicon (Figure $8 \mathrm{~B}$ ), while under unpolarized illumination only a single band is observed, the vertical dipole mode is both red-shifted and 100 -fold more intense than the horizontal mode. For a nanoparticle on a gold film (Figure 8A), both modes observed in experiment are modes excited almost exclusively by the p-polarized component. Thus the higher energy mode is most likely a higher order mode possessing dipole character by virtue of sphere mode hybridization mediated by the film. ${ }^{64,65}$ Response to the s-polarized component consists of a weakly scattering mode at 530 $\mathrm{nm}$ and an even weaker mode at $\sim 563 \mathrm{~nm}$. In nanoparticle-film systems where the uncoupled particle modes are all lower energy than the interacting continuum of the film, theoretical studies indicate that hybridized particle modes retain their spectral order as coupling to a substrate is increased, such that the longest wavelength mode of a given azimuthal character will be the mode with the greatest dipolar character. ${ }^{64,65,67}$ In the gold nanoparticle-gold film system investigated here, it appears that, while s-wave excited modes scatter too weakly to be readily detected, at least one higher order mode can be observed.

The dominance of the spectrum by the brightest vertically oriented mode contributes to the spectral control offered by the simple geometry of a particle on a planar film. The match between representative (mean intensity) single particle Rayleigh spectra and simulated spectra provides strong evidence that the plasmonic properties of these junction-bearing substrates are well-controlled. Nonetheless, a better understanding of the robustness of the system requires con- 
sideration of the ensemble of individual particle spectra. Thus, a full set of single particle Rayleigh scattering spectra from each substrate are displayed (Figure $9 A-C)$. Spectra of particles on each surface are remarkably reproducible with respect to both intensity and peak position. Variations in intensity relative to mean intensity are 31,18 , and $25 \%$ for particles on gold, silicon, and quartz, respectively. Standard deviations in peak wavelength are 7 and $6 \mathrm{~nm}$ for particles on gold and silicon and $1.6 \mathrm{~nm}$ for particles on quartz.

To gain insight into the geometric origins of the observed spectral variations, scattering spectra simulations were performed for particles of variable size or variable separation from the three substrates. On all surfaces, scattering intensity depends strongly upon particle size (Figure 9D-F). For size variations within one standard deviation, variation in intensity scales nearly linearly with the fourth power of the size (Supporting Information 5). The dependence of scattering intensity on size for particles on a surface thus matches that of spherical particles in homogeneous media. The sensitivity of band position to size varied significantly among the substrates. Variation in peak position with $a+\sigma /-\sigma$ change in particle size is $+11 /-8,+4 /-4$, and $<1 \mathrm{~nm}$ for particles on gold, silicon, and quartz, respectively. Thus, antenna plasmons of particles of variable size on the more highly polarizable surfaces display positively correlated dispersion in scattering intensity and peak wavelength. On these substrates, positive correlation between variation in intensity and band position is the signature of size dispersion. The result is expected to be general except when confounded by wavelengthdependent interband transitions.

Gap width plays a key role in determining plasmon resonance and a critical role in field intensity in a metal-insulator-metal gap. We are interested in both the signatures of gap width variation and sensitivity of simulation results to errors in measurements of thicknesses of spacer layers. To test the sensitivity of the scattering to variations in polyelectrolyte and/or silica thickness, spectra were simulated for nanoparticles separated from surfaces by dielectric layers with thicknesses different from the thicknesses derived from ellipsometry. Representative simulated spectra are shown in Figure $9 \mathrm{G}-\mathrm{I}$. On gold and silicon a $1 \AA$ increase in thickness of the polyelectrolyte spacer layer results, respectively, in a 4 or $0.7 \mathrm{~nm}$ blue shift in the nanoparticle scattering band position. On quartz, low contrast of the polyelectrolyte relative to quartz renders the spectrum insensitive to changes in thickness of the PE layer. The sensitivities to dielectric layer thickness on gold and silicon are consistent with the expectation that plasmons in strongly coupled structures will have resonances highly sensitive to gap width. While intensity also is sensitive to gap width, the change in intensity relative to the change in band position is much less if
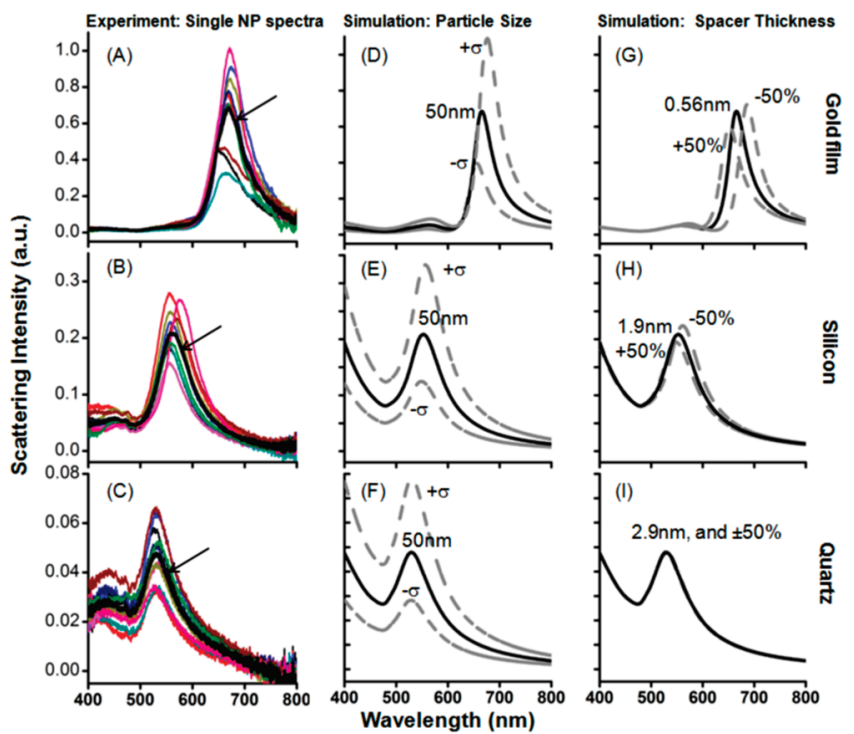

Figure 9. Single particle scattering spectra and spectral simulations of gold nanoparticles on three surfaces. $(A-C)$ Rayleigh scattering from single gold nanoparticles functionalized with Cy5-modified oligonucleotide on a polyelectrolyte layer on (A) a gold film, (B) a silicon wafer, and (C) a quartz wafer. Single particle spectra are colored. The average of the single particle spectra is identified by an arrow and shown in black. (D-F) Simulated spectra for nanoparticles of mean size and $+\sigma /-\sigma$ variations. (G-I) Simulated spectra for nanoparticles on the three surfaces with spacer layers of varying thickness. For the $(G)$ gold and (I) quartz surfaces, the layer thickness varied is the thickness of the polyelectrolyte layer. For the $(\mathrm{H})$ silicon surface, the thickness varied is the combined thickness of the polyelectrolyte and oxide layers. For all simulations, the nominal thickness value is the thickness measured by ellipsometry. Note that, in simulations, gap widths are larger than spacer thicknesses by the thickness, $0.3 \mathrm{~nm}$, assigned to the nanoparticle dielectric shell.

due to variation in gap width than if due to variation in particle size.

Knowledge of the sensitivities of antenna scattering to particle size and spacer thickness leads to hypotheses about the geometric basis for both variations and invariance observed in the single antenna spectra. Minimal variation in peak position among the antenna spectra of particles on quartz (Figure 9C) is explained by the insensitivity of the band position to either particle size or particle-surface separation displayed in the simulations (Figure 9F,I). For the weakly coupled gold-quartz system (Figure 9C), the invariance of peak position further indicates low dispersion in particle shape. As the intensity of scattering from gold particles on quartz is insensitive to the thickness of the polyelectrolyte layer, we interpret the observed variation in intensity as a manifestation of dispersion in particle size. From the sensitivities displayed in simulation, the observed variation of $25 \%$ in intensity corresponds to a variation in particle size of $\sim 6 \%$. Scattering spectra from gold nanoparticles on silicon (Figure 9B) display a similar variation in intensity and also variation in band position. As the band position for nanoparticles on silicon varies only slightly with size dispersion, the observed variation in band position is most likely a manifestation of variation in particle-surface separation. 
This hypothesis is strengthened by the minimal variation in band position observed for particles on the most weakly interacting surface, quartz, as low dispersion in resonance wavelength rules against the alternative explanation, heterogeneity in particle shape. On silicon, nanoparticle-silicon separation is determined by the combined thicknesses of the optically similar polyelectrolyte and oxide layer, and variation in gap width cannot be attributed to a specific layer. Correlation of peak intensity and band position observed among single particle spectra of gold particles on a gold film (Figure $9 \mathrm{~A}$ ) is displayed also in simulations of spectra of particles dispersed in size. The variation in intensity is consistent with the size distribution displayed by the nanoparticles on quartz. In the spectra of gold particles on gold film, the correlation between intensity, l, and peak location, $\lambda^{*}$, is somewhat scrambled relative to what would be expected from size variation alone. This suggests that the single nanoparticle spectra may reveal the variation among nanoantenna gap widths. From comparison with simulations of the gap width dependence of Au nanoparticles on gold (Figure 9G), scrambling of the $\lambda^{*}-/$ correlation in the single structure spectra is consistent with a modest $(\sim 12 \%)$ variation in spacing between the nanoparticles and the gold film (Supporting Information 6).

The resonant Raman scattering enhancement provided by the coupling of the nanoparticle to the gold film enables not only Raman imaging but also spectral collection from a small number of molecules in gaps between a small number of nanoparticles and the film. Having determined previously a Raman cross section of $\mathrm{Cy} 5,{ }^{31}$ it should be possible to use the molecules to probe gap fields. As the Raman scattered signals are too weak to allow collection of a single molecule spectrum, ensemble data may nonetheless be useful if the variation in gap widths and, thus, gap fields is not large. In the PE coupled system, variations in gap width are remarkably small. Thus variation in gap fields and electromagnetic SERS enhancement also will be unusually small. If Raman signals are strong enough to allow Raman bands to be collected from several molecules in gaps, the integrated bands may provide meaningful estimates of antenna gap fields. In our experimental system, the excitation power density, collection angle (defined by NA), number of nanoparticles illuminated, and density of $\mathrm{Cy} 5$ on each nanoparticle are well-defined. Prior understanding of the tight confinement of high fields within a particle-film gap ${ }^{27,68,69}$ combined with low Cy5 coverage ( 1 per $4 \mathrm{~nm}^{2}$ ) collectively suggests that a SERS enhancement factor for molecules in the gap may be determined under the assumption that Raman scattering is from one molecule per particle. Using the Raman cross section determined previously (order $10^{-25} \mathrm{~cm}^{2} / \mathrm{sr}$ after correction for electromagnetic enhancement) for the $1191 \mathrm{~cm}^{-1}$ Cy5 Raman band $\left(\lambda_{0}=632.8 \mathrm{~nm}\right)$ from the functionalized nanoparticles in bulk ${ }^{31}$ and the efficiency of collection of the scattering sig- nal from a particle on a film, we obtain a total apparent Raman cross section of $1 \times 10^{-17} \mathrm{~cm}^{2}$ from a spectrum collected from three nanoantenna (Supporting Information 7). Assuming isotropic scattering for the solution phase Raman scattering, the electromagnetic SERS enhancement in the gold particle-polyelectrolyte-gold film system is estimated to be $8 \times 10^{6}$. This electromagnetic SERS enhancement factor is surprisingly similar to a mean gap enhancement factor reported for a much larger number of molecules in a silver dimer gap $\left(2 \times 10^{7}\right)^{70}$ and a peak enhancement factor for much smaller silver nanoparticles on a thicker gold film. ${ }^{27}$ However, we have neglected unknown effects of differences in on-state lifetimes for molecules in electric fields of unlike strength.

\section{CONCLUSION}

We have demonstrated that nanoantennas composed of gold nanoparticles on spacer layers on diverse surfaces radiate light at frequencies that differ significantly and depend predictably upon the complex permittivities of the materials of which the surface is composed. Plasmon band position sensitivity to permittivity has been observed previously when metal nanoparticles are deposited on dielectric films. ${ }^{39,40}$ The wavelengths of the scattering resonance of the nanoantenna, however, are very different on gold and silicon despite similar permittivity magnitudes and differences in gap width insufficient to explain the spectral variation. The electronic character of the materials, therefore, plays an important role; at optical frequencies, only the metal has a negative permittivity and supports surface plasmon waves, whereas the response of silicon is dominated by electrons that are bound. While we have not tested the match of simulation to resonance of a metal particle on a metal film under TIR excitation, the implemented treatment of reflection captures intrinsically not only coupling among localized plasmon modes but also the interplay between localized modes and radially propagating surface waves.

It further has been shown that, for the particle on film geometry, not only band positions but also Rayleigh scattering intensities are both enhanced and wellcontrolled. On silicon and a gold film, scattering from the brightest mode of a $50 \mathrm{~nm}$ gold nanoparticle is enhanced by approximately 5 - and 25 -fold respectively, relative to scattering from a free nanoparticle dipole mode. While we collect scattering primarily from vertical modes that do not scatter particularly efficiently into even a high NA objective, enhancement of vertical modes by the substrates allows VED scattering from single particles to be readily detected. From simulations and Rayleigh scattering characterization of single nanoantennas on all three surfaces, the intensity of VED scattering is found to depend not only upon the composition of the surface but also sensitively upon nanoparticle size. 
In junction-bearing near-field-enhancing nanostructured substrates, the spectral signatures of variations in geometry are in general not unique. We therefore have used simulations to determine the distinctiveness of the plasmonic signatures of geometric parameters of key importance to this system. For particles on surfaces that shift the primary plasmon band position to the red region, where surface plasmon band positions of gold nanostructures are highly sensitive to perturbations in the resonance conditions, intensity variations associated with dispersion in particle size are accompanied by correlated small shifts in band position. Gap width variations in contrast are manifest primarily as variation in peak position. Whereas in many coupled structures band position variation reflects variation in shape and orientation, as well as size and junction width, in the particle-surface system studied here, deviation from the particle-size-dependent $I-\lambda^{*}$ correlation provides a measure of variation in gap width. This assignment is made possible by the insensitivity of the scattered signal to other geometric variables such as shape dispersion by virtue of the vertical orientation of the brightest plasmon mode. The observation is of relevance to quantitative detection of SERS (and SERRS), as variations in SERS enhancement from nanostructure to nanostructure will be amenable to correction if single structure spectral data can be used to identify gap widths and gap fields.

From imaging, we find that the scattering is dominated by a vertically oriented plasmon excitation when the nanoparticle is deposited on either highly polarizable material, gold or silicon. For the Raman-active substrate examined in this work, Raman images, like Rayleigh images, are doughnut-shaped, indicating that the directionality of SERRS is controlled by the orientation of the radiative plasmon mode. The result extends control of polarization of molecule emission previously ob- served for fluorescence ${ }^{47,48}$ to Raman scattering. In the Raman-active system composed of nanoparticles on a gold film, blinking of the Raman images of cyaninefunctionalized gold nanoparticles was observed. SERRS was not observed from particles on surfaces other than gold. However, the polarization control provided by the metal nanoparticle on silicon system suggests that nanoparticles will play a role in directing light in integrated optoelectronic systems.

Polarization control is critical also in both fundamental studies of plasmons and their interactions with molecules and in applications that require quantitative measurements of SE(R)RS. Control both of orientation and of gap width allows not only excitation and emission but also efficiency of collection to be controlled. We observe not only control of antenna surface plasmon resonance intensity and band position but also control by the substrate of the polarization of both Rayleigh and Raman emission, which enables the fraction of emitted light collected to be controlled. On a gold film, near-field enhancement and radiative rates are sufficiently large to allow Raman scattering images and spectra to be collected from a small number of blinking molecules. The few molecule measurements allow an electromagnetic SERS enhancement factor $\left(8 \times 10^{6}\right)$ to be derived using a Raman cross section determined previously for Cy5 in a weaker field. ${ }^{31}$ While the enhancement value is in the same range as values determined for junction-bearing noble metal nanostructures investigated by others, ${ }^{70}$ differences in on-state lifetimes in fields of different strengths will significantly affect enhancement estimates. We nonetheless suspect that the brightness of nanoparticle antenna and their sensitivity to surface permittivity will enable nanoparticle antennas to serve as reporters of both surface microstructure and the properties of surface molecules.

\section{METHODS}

Sample Preparation. Gold particles (BBI, mean size $50.8 \mathrm{~nm}$, maximum standard deviation $4 \mathrm{~nm}$ ) were functionalized with Cy5-modified 22-base oligonucleotides terminated with a $5^{\prime}$ thiol. (IDT DNA) The cyanine species, Cy5, was adjacent to the terminal alkane thiol. The adjacent 10 bases (adenines) have affinity for gold. The absorbance of the nanoparticles and fluorescence of the oligonucleotide solution and supernatants from successive rinses were recorded and used to quantify the concentrations of particles, excess cyanine, and immobilized cyanine and the ratio of concentrations of immobilized cyanine and particles. The coverage of oligonucleotides was $\sim 2000$ strands per nanoparticle (Supporting Information 8). Detailed functionalization procedures can be found in previous reports. ${ }^{31,71}$

The quartz substrate was cut from a wafer (SQI) and cleaned with piranha solution $\left(\mathrm{H}_{2} \mathrm{SO}_{4} / \mathrm{H}_{2} \mathrm{O}_{2}=3: 1\right)$. The semiconductor substrates were $\mathrm{p}$-type silicon wafers; the silicon was cleaned using acetone. The gold film on quartz was prepared by depositing a $5 \mathrm{~nm}$ chromium adhesion layer and a $45 \mathrm{~nm}$ gold thin film using e-beam evaporation (CHA Industries). Cationic polyelectrolyte (PE) was deposited on all three surfaces using the same selflimiting procedure. ${ }^{57}$ Substrates were immersed in a 0.003 mols of monomer/L solution of poly(allylamine) hydrochloride $(\mathrm{PAH}) / 1 \mathrm{M} \mathrm{NaCl}$ for $30 \mathrm{~min}$, immersed in DI water for $1 \mathrm{~min}$, rinsed with $\mathrm{DI}$ water, and dried under high purity nitrogen. A $100 \mu \mathrm{L}$ drop of diluted functionalized gold particle solution $\left(\sim 10^{-11} \mathrm{M}\right)$ was placed on the PE surface of each surface for 1 min, rinsed with DI water, and dried with nitrogen.

Thin Film Measurement. Select layer indices and thicknesses were characterized by ellipsometry (J.A. Woollam M-88). Amplitude ratios and phase shifts were acquired at incident (and collection) angles of 65,70 , and $75^{\circ}$ with wavelength scanning from 277.5 to $763 \mathrm{~nm}$. Indices of refraction for the gold thin film and the thickness of the native oxide on silicon were determined prior to deposition of the polyelectrolyte, $\mathrm{PAH}$. The refractive index of PAH was determined after deposition by fitting parameters in the Cauchy expression for normal dispersion to reflectivity data collected from PAH on gold (Supporting Information 9) while simultaneously determining the thickness of the PAH thin film. The measurement and data analysis were performed using the software WVASE32. The refractive indices of silicon and quartz are from the literature. ${ }^{72,73}$

Dark-Field and Raman Image Setup. All images, Rayleigh spectra, and some Raman spectra were acquired in a custom micro- 
scope equipped with two output ports; typically, output from one port was relayed to a spectrograph, and output from the other was used for imaging. A commercial digital camera ( $\mathrm{Ni}$ kon D90) captured color dark-field images. Raman images and gray level images of elastic scattering were recorded on a CCD (Photometrics CoolSnap ES). Signals were collected through a $50 \times$ (Nikon CF Plan NA 0.55) or $100 \times$ (Nikon CF Plan NA 0.9) objective lens. A long pass filter (Semrock LP02-633RU-25) was inserted between the objective lens and camera to remove the laser line when collecting Raman signals.

Spectrum Acquisition Setup. For Raman and Rayleigh scattering spectra, the scattering light was collected through the other port of the microscope and then relayed to a TE-cooled CCD (Photometric CoolSnap HQ). A pinhole is usually added to reduce background noise. For acquisition of Raman spectra, a high-resolution grating (1800 grooves) was used. For Rayleigh scattering, a lower resolution grating (50 grooves) centered at $650 \mathrm{~nm}$ and spanning from 300 to $900 \mathrm{~nm}$ was used to obtain the broad-band spectrum. Raman spectra $\left(\lambda_{0}=632.8 \mathrm{~nm}\right)$ also were collected using a commercial Raman spectrometer (Horiba LabRam ARAMIS) equipped with an $8 \mathrm{~mW}$ HeNe laser and 100X objective lens.

Excitation Methods. For Rayleigh scattering spectra or images, the light source was a $75 \mathrm{~W}$ xenon lamp (OSRAM XBO); white light was directed to the nanoparticles through the objective lens. To acquire Raman signals, $30 \mathrm{~mW}$ HeNe laser was used to excite the particles directly from the side or introduced through a prism at a $45^{\circ}$ angle that led to total internal reflection. Side ex citation allowed illumination of opaque substrates, and polarization of the incident laser was easily controlled by positioning a polarizer in front of the sample. To acquire the highest resolution images and characterize the point spread functions, TIR excitation of the particles on the gold film was used. TIR excitation allowed collection of low angle scattering through a highmagnification objective $(100 \times)$ with a very small working distance. In this work, spectra and images were collected in the TIR configuration from nanoparticles on a gold film.

Simulations. Scattering by the antennas into a cone determined by the objective was simulated using the method of Bobbert and Vlieger ${ }^{66}$ (BV). The BV method describes scattering from spheres on planar surfaces and is a multipolar generalization of Sommerfeld's method for evaluating scattering from a dipole on a planar surface. ${ }^{74}$ We used the implementation of Germer, ${ }^{35,75}$ which incorporates a dielectric shell on the particle and a multilayered planar surface. Scattering spectra were computed initially for a series of particle-surface geometries in which a planar spacer layer atop the PE layer was assigned a width of decreasing thickness. Subsequently, the spacer thickness was assigned to the nanoparticle as a shell. In our system, this shell corresponds to space partially occupied by the modified oligonucleotides that cap the gold nanoparticles. As the properties of the capping layer in air were not known, in our simulations, the shell was assigned a thickness, $0.3 \mathrm{~nm}$, and effective index, 1.22, consistent with the Rayleigh data. For sensitivity studies, the optical constants and dielectric functions used to describe the gold, silicon, and quartz were from Johnson and Christy, ${ }^{56}$ Green and Keevers, ${ }^{76}$ and Palik, ${ }^{72}$ respectively. For comparison with experiment, optical constants and dielectric functions from the ellipsometric measurements were used.

Acknowledgment. A.L. acknowledges support of the NSF (DMR-0706397). S.-Y.C. thanks B.N. Hsu and Y.Y. Lin for experimental suggestions and H.K. Yuan for useful discussion.

Supporting Information Available: (1) Temporal variation in Raman scattering from single nanoparticle junctions; (2) Cy5 Raman control; (3) simulation method; (4) effect of oxide thickness on NP-silicon coupling; (5) simulation results: intensity-particle size scaling; (6) estimation of spacer thickness variation; (7) estimation of Cy5 Raman scattering cross section and electromagnetic (EM) enhancement; (8) determination of coverage of Cy5modified oligonucleotides on particles; (9) parametric form of wavelength-dependent refractive index model used in ellipsometric characterization of PAH. This material is available free of charge via the Internet at http://pubs.acs.org.

\section{REFERENCES AND NOTES}

1. Kneipp, K.; Wang, Y.; Kneipp, H.; Perelman, L. T.; Itzkan, I.; Dasari, R.; Feld, M. S. Single Molecule Detection Using Surface-Enhanced Raman Scattering (SERS). Phys. Rev. Lett. 1997, 78, 1667-1670.

2. Nie, S. M.; Emery, S. R. Probing Single Molecules and Single Nanoparticles by Surface-Enhanced Raman Scattering. Science 1997, 275, 1102-1106.

3. Michaels, A. M.; Nirmal, M.; Brus, L. E. Surface Enhanced Raman Spectroscopy of Individual Rhodamine $6 \mathrm{~g}$ Molecules on Large Ag Nanocrystals. J. Am. Chem. Soc. 1999, 121, 9932-9939.

4. Xu, H. X.; Bjerneld, E. J.; Kall, M.; Borjesson, L. Spectroscopy of Single Hemoglobin Molecules by Surface Enhanced Raman Scattering. Phys. Rev. Lett. 1999, 83, 4357-4360.

5. Graham, D.; Mallinder, B. J.; Smith, W. E. Surface-Enhanced Resonance Raman Scattering as a Novel Method of DNA Discrimination. Angew. Chem., Int. Ed. 2000, 39, 1061-1063.

6. Ausman, L. K.; Schatz, G. C. On the Importance of Incorporating Dipole Reradiation in the Modeling of Surface Enhanced Raman Scattering from Spheres. J. Chem. Phys. 2009, 131, 084708-1-084708-10.

7. Kerker, M.; Wang, D.-S.; Chew, H. Surface Enhanced Raman Scattering (SERS) by Molecules Adsorbed at Spherical Particles: Errata. Appl. Opt. 1980, 19, 4159-4174.

8. Creighton, J. A.; Blatchford, C. G.; Albrecht, M. G. Plasma Resonance Enhancement of Raman-Scattering by Pyridine Adsorbed on Silver or Gold Sol Particles of Size Comparable to the Excitation Wavelength. J. Chem. Soc., Faraday Trans. 2 1979, 75, 790-798.

9. Freeman, R. G.; Grabar, K. C.; Allison, K. J.; Bright, R. M.; Davis, J. A.; Guthrie, A. P.; Hommer, M. B.; Jackson, M. A.; Smith, P. C.; Walter, D. G.; et al. Self-Assembled Metal Colloid Monolayers - An Approach to SERS Substrates. Science 1995, 267, 1629-1632.

10. Oldenburg, S. J.; Westcott, S. L.; Averitt, R. D.; Halas, N. J. Surface Enhanced Raman Scattering in the Near Infrared Using Metal Nanoshell Substrates. J. Chem. Phys. 1999, 111, 4729-4735.

11. Tian, Z. Q.; Ren, B.; Wu, D. Y. Surface-Enhanced Raman Scattering: From Noble to Transition Metals and from Rough Surfaces to Ordered Nanostructures. J. Phys. Chem. B 2002, 106, 9463-9483.

12. Nikoobakht, B.; El-Sayed, M. A. Surface-Enhanced Raman Scattering Studies on Aggregated Gold Nanorods. J. Phys. Chem. A 2003, 107, 3372-3378.

13. McLellan, J. M.; Xiong, Y. J.; Hu, M.; Xia, Y. N. SurfaceEnhanced Raman Scattering of 4-Mercaptopyridine on Thin Films of Nanoscale Pd Cubes, Boxes, and Cages. Chem. Phys. Lett. 2006, 417, 230-234.

14. Ruan, C. M.; Eres, G.; Wang, W.; Zhang, Z. Y.; Gu, B. H. Controlled Fabrication of Nanopillar Arrays as Active Substrates for Surface-Enhanced Raman Spectroscopy. Langmuir 2007, 23, 5757-5760.

15. Driskell, J. D.; Shanmukh, S.; Liu, Y.; Chaney, S. B.; Tang, X. J.; Zhao, Y. P.; Dluhy, R. A. The Use of Aligned Silver Nanorod Arrays Prepared by Oblique Angle Deposition as Surface Enhanced Raman Scattering Substrates. J. Phys. Chem. C 2008, 112, 895-901.

16. Yu, Q. M.; Guan, P.; Qin, D.; Golden, G.; Wallace, P. M. Inverted Size-Dependence of Surface-Enhanced Raman Scattering on Gold Nanohole and Nanodisk Arrays. Nano Lett. 2008, 8, 1923-1928.

17. Khoury, C. G.; Vo-Dinh, T. Gold Nanostars for SurfaceEnhanced Raman Scattering: Synthesis, Characterization and Optimization. J. Phys. Chem. C 2008, 112, 18849-18859.

18. Olk, P.; Renger, J.; Hartling, T.; Wenzel, M. T.; Eng, L. M. Two Particle Enhanced Nano Raman Microscopy and Spectroscopy. Nano Lett. 2007, 7, 1736-1740.

19. Ringler, M.; Klar, T. A.; Schwemer, A.; Susha, A. S.; Stehr, J.; Raschke, G.; Funk, S.; Borowski, M.; Nichtl, A.; Kurzinger, K.; et al. Moving Nanoparticies with Raman Scattering. Nano Lett. 2007, 7, 2753-2757.

20. Zheng, J. W.; Zhou, Y. G.; Li, X. W.; Ji, L.; Lu, T. H.; Gu, R. A. 
Surface-Enhanced Raman Scattering of 4Aminothiophenol in Assemblies of Nanosized Particles and the Macroscopic Surface of Silver. Langmuir 2003, 19, 632-636.

21. Daniels, J. K.; Chumanov, G. Nanoparticle-Mirror Sandwich Substrates for Surface-Enhanced Raman Scattering. J. Phys. Chem. B 2005, 109, 17936-17942.

22. Kim, K.; Yoon, J. K. Raman Scattering of 4Aminobenzenethiol Sandwiched between $\mathrm{Ag} / \mathrm{Au}$ Nanoparticle and Macroscopically Smooth Au Substrate. J. Phys. Chem. B 2005, 109, 20731-20736.

23. Orendorff, C. J.; Gole, A.; Sau, T. K.; Murphy, C. J. SurfaceEnhanced Raman Spectroscopy of Self-Assembled Monolayers: Sandwich Architecture and Nanoparticle Shape Dependence. Anal. Chem. 2005, 77, 3261-3266.

24. Driskell, J. D.; Lipert, R. J.; Porter, M. D. Labeled Gold Nanoparticles Immobilized at Smooth Metallic Substrates: Systematic Investigation of Surface Plasmon Resonance and Surface-Enhanced Raman Scattering. J. Phys. Chem. B 2006, 110, 17444-17451.

25. Braun, G.; Lee, S. J.; Dante, M.; Nguyen, T. Q.; Moskovits, M.; Reich, N. Surface-Enhanced Raman Spectroscopy for DNA Detection by Nanoparticle Assembly onto Smooth Metal Films. J. Am. Chem. Soc. 2007, 129, 6378-6379.

26. Park, W. H.; Ahn, S. H.; Kim, Z. H. Surface-Enhanced Raman Scattering from a Single Nanoparticle-Plane Junction. ChemPhysChem 2008, 9, 2491-2494.

27. Tang, J.; Ponizovskaya, E. V.; Bratkovsky, A. M.; Stewart, D. R.; Li, Z. Y.; Williams, R. S. Metallic Nanocrystals near Ultrasmooth Metallic Films for Surface-Enhanced Raman Scattering Application. Nanotechnology 2008, 19, 415702.

28. Ikeda, K.; Sato, J.; Fujimoto, N.; Hayazawa, N.; Kawata, S.; Uosaki, K. Plasmonic Enhancement of Raman Scattering on Non-SERS-Active Platinum Substrates. J. Phys. Chem. C 2009, 113, 11816-11821.

29. Yoon, J. K.; Kim, K.; Shin, K. S. Raman Scattering of 4Aminobenzenethiol Sandwiched between $\mathrm{Au}$ Nanoparticles and a Macroscopically Smooth Au Substrate: Effect of Size of Au Nanoparticles. J. Phys. Chem. C 2009, 113, 1769-1774.

30. Wokaun, A.; Lutz, H. P.; King, A. P.; Wild, U. P.; Ernst, R. R. Energy-Transfer in Surface Enhanced Luminescence. J. Chem. Phys. 1983, 79, 509-514.

31. Chen, S. Y.; Lazarides, A. A. Quantitative Amplification of Cy5 SERS in 'Warm Spots' Created by Plasmonic Coupling in Nanoparticle Assemblies of Controlled Structure. J. Phys. Chem. C 2009, 113, 12167-12175.

32. Mock, J. J.; Hill, R. T.; Degiron, A.; Zauscher, S.; Chilkoti, A.; Smith, D. R. Distance-Dependent Plasmon Resonant Coupling between a Gold Nanoparticle and Gold Film. Nano Lett. 2008, 8, 2245-2252.

33. Stuart, H. R.; Hall, D. G. Island Size Effects in NanoparticleEnhanced Photodetectors. Appl. Phys. Lett. 1998, 73, 38153817.

34. Kume, T.; Hayashi, S.; Yamamoto, K. Light Emission from Surface Plasmon Polaritons Mediated by Metallic Fine Particles. Phys. Rev. B 1997, 55, 4774-4782.

35. Kim, J. H.; Ehrman, S. H.; Mulholland, G. W.; Germer, T. A. Polarized Light Scattering by Dielectric and Metallic Spheres on Silicon Wafers. Appl. Opt. 2002, 41, 5405-5412.

36. Hu, M.; Ghoshal, A.; Marquez, M.; Kik, P. G. Single Particle Spectroscopy Study of Metal-Film-Induced Tuning of Silver Nanoparticle Plasmon Resonances. J. Phys. Chem. C 2010, 114, 7509-7514

37. Okamoto, T.; Yamaguchi, I. Optical Absorption Study of the Surface Plasmon Resonance in Gold Nanoparticles Immobilized onto a Gold Substrate by Self-Assembly Technique. J. Phys. Chem. B 2003, 107, 10321-10324.

38. You, Y. M.; Du, C. L.; Ma, Y.; Kasim, J.; Yu, T.; Shen, Z. X. Effect of Near-Field Coupling on Far-Field Inelastic Scattering Imaging of Gold Nanoparticles. Nanotechnology 2008, $19,5$.

39. Knight, M. W.; Wu, Y. P.; Lassiter, J. B.; Nordlander, P.; Halas,
N. J. Substrates Matter: Influence of an Adjacent Dielectric on an Individual Plasmonic Nanoparticle. Nano Lett. 2009, 9, 2188-2192.

40. Du, C. L.; You, Y. M.; Johnson, K.; Hu, H. L.; Zhang, X. J.; Shen, Z. X. Near-Field Coupling Effect between Individual Au Nanospheres and Their Supporting $\mathrm{SiO}_{2} / \mathrm{Si}$ Substrate. Plasmonics 2010, 5, 105-109.

41. Knight, M. W.; Fan, J.; Capasso, F.; Halas, N. J. Influence of Excitation and Collection Geometry on the Dark Field Spectra of Individual Plasmonic Nanostructures. Opt. Express 2010, 18, 2579-2587.

42. Rechberger, W.; Hohenau, A.; Leitner, A.; Krenn, J. R.; Lamprecht, B.; Aussenegg, F. R. Optical Properties of Two Interacting Gold Nanoparticles. Opt. Commun. 2003, 220, 137-141.

43. Su, K. H.; Wei, Q. H.; Zhang, X.; Mock, J. J.; Smith, D. R.; Schultz, S. Interparticle Coupling Effects on Plasmon Resonances of Nanogold Particles. Nano Lett. 2003, 3, 1087-1090.

44. Fromm, D. P.; Sundaramurthy, A.; Schuck, P. J.; Kino, G.; Moerner, W. E. Gap-Dependent Optical Coupling of Single "Bowtie" Nanoantennas Resonant in the Visible. Nano Lett. 2004, 4, 957-961.

45. Atay, T.; Song, J. H.; Nurmikko, A. V. Strongly Interacting Plasmon Nanoparticle Pairs: From Dipole-Dipole Interaction to Conductively Coupled Regime. Nano Lett. 2004, 4, 1627-1631.

46. Muhlschlegel, P.; Eisler, H. J.; Martin, O. J. F.; Hecht, B.; Pohl, D. W. Resonant Optical Antennas. Science 2005, 308, 1607-1609.

47. Moerland, R. J.; Taminiau, T. H.; Novotny, L.; Van Hulst, N. F.; Kuipers, L. Reversible Polarization Control of Single Photon Emission. Nano Lett. 2008, 8, 606-610.

48. Taminiau, T. H.; Stefani, F. D.; Segerink, F. B.; Van Hulst, N. F. Optical Antennas Direct Single-Molecule Emission. Nat. Photonics 2008, 2, 234-237.

49. Xu, H. X.; Kall, M. Polarization-Dependent SurfaceEnhanced Raman Spectroscopy of Isolated Silver Nanoaggregates. ChemPhysChem 2003, 4, 1001-1005.

50. Farahani, J. N.; Pohl, D. W.; Eisler, H. J.; Hecht, B. Single Quantum Dot Coupled to a Scanning Optical Antenna: A Tunable Superemitter. Phys. Rev. Lett. 2005, 95, 017402.

51. Muskens, O. L.; Giannini, V.; Sanchez-Gil, J. A.; Rivas, J. G. Strong Enhancement of the Radiative Decay Rate of Emitters by Single Plasmonic Nanoantennas. Nano Lett. 2007, 7, 2871-2875.

52. Zhang, J.; Fu, Y.; Chowdhury, M. H.; Lakowicz, J. R. MetalEnhanced Single-Molecule Fluorescence on Silver Particle Monomer and Dimer: Coupling Effect between Metal Particles. Nano Lett. 2007, 7, 2101-2107.

53. Bouhelier, A.; Beversluis, M. R.; Novotny, L. Characterization of Nanoplasmonic Structures by Locally Excited Photoluminescence. Appl. Phys. Lett. 2003, 83, 5041-5043.

54. Schuck, P. J.; Fromm, D. P.; Sundaramurthy, A.; Kino, G. S.; Moerner, W. E. Improving the Mismatch between Light and Nanoscale Objects with Gold Bowtie Nanoantennas. Phys. Rev. Lett. 2005, 94, 017402.

55. Ghenuche, P.; Cherukulappurath, S.; Taminiau, T. H.; van Hulst, N. F.; Quidant, R. Spectroscopic Mode Mapping of Resonant Plasmon Nanoantennas. Phys. Rev. Lett. 2008, $101,116805$.

56. Johnson, P. B.; Christy, R. W. Optical-Constants of NobleMetals. Phys. Rev. B 1972, 6, 4370-4379.

57. Ramsden, J. J.; Lvov, Y. M.; Decher, G. Determination of Optical-Constants of Molecular Films Assembled via Alternate Polyion Adsorption. Thin Solid Films 1995, 254, 246-251.

58. Novotny, L.; Hecht, B. Principles of Nano-Optics; Cambridge University Press: Cambridge, 2006.

59. Anger, P.; Bharadwaj, P.; Novotny, L. Enhancement and Quenching of Single-Molecule Fluorescence. Phys. Rev. Lett. 2006, 96, 113002-1-113002-4.

60. Neuhauser, D.; Lopata, K. Molecular Nanopolaritonics: Cross Manipulation of Near-Field Plasmons and Molecules. 
I. Theory and Application to Junction Control. J. Chem. Phys. 2007, 127, 154715-1-154715-10.

61. Zhao, J.; Sherry, L. J.; Schatz, G. C.; Van Duyne, R. P. Molecular Plasmonics: Chromophore-Plasmon Coupling and Single-Particle Nanosensors. IEEE J. Sel. Top. Quantum Electron. 2008, 14, 1418-1429.

62. Miroshnichenko, A. E.; Flach, S.; Kivshar, Y. S. Fano Resonances in Nanoscale Structures. Rev. Mod. Phys. 2010, 82, 2257-2298.

63. Raether, H. Surface Plasmons on Smooth and Rough Surfaces and on Gratings; Springer-Verlag: Berlin, 1988.

64. Le, F.; Lwin, N. Z.; Steele, J. M.; Kall, M.; Halas, N. J.; Nordlander, P. Plasmons in the Metallic Nanoparticle-Film System as a Tunable Impurity Problem. Nano Lett. 2005, 5, 2009-2013.

65. Nordlander, P.; Prodan, E. Plasmon Hybridization in Nanoparticles near Metallic Surfaces. Nano Lett. 2004, 4, 2209-2213.

66. Bobbert, P. A.; Vlieger, J. Light-Scattering by a Sphere on a Substrate. Physica A 1986, 137, 209-242.

67. Wu, Y. P.; Nordlander, P. Finite-Difference Time-Domain Modeling of the Optical Properties of Nanoparticles near Dielectric Substrates. J. Phys. Chem. C 2010, 114, 7302-7307.

68. Aravind, P. K.; Metiu, H. The Effects of the Interaction between Resonances in the Electromagnetic Response of a Sphere-Plane Structure-Applications to Surface Enhanced Spectroscopy. Surf. Sci. 1983, 124, 506-528.

69. Nordlander, P.; Le, F. Plasmonic Structure and Electromagnetic Field Enhancements in the Metallic Nanoparticle-Film System. Appl. Phys. B 2006, 84, 35-41.

70. Li, W. Y.; Camargo, P. H. C.; Lu, X. M.; Xia, Y. N. Dimers of Silver Nanospheres: Facile Synthesis and Their Use as Hot Spots for Surface-Enhanced Raman Scattering. Nano Lett. 2009, 9, 485-490.

71. Cao, Y. W. C.; Jin, R. C.; Mirkin, C. A. Nanoparticles with Raman Spectroscopic Fingerprints for DNA and RNA Detection. Science 2002, 297, 1536-1540.

72. Palik, E. D. Handbook of Optical Constants of Solids; Academic Press: Orlando, FL, 1985.

73. Herzinger, C. M.; Johs, B.; McGahan, W. A.; Woollam, J. A.; Paulson, W. Ellipsometric Determination of Optical Constants for Silicon and Thermally Grown Silicon Dioxide via a Multi-Sample, Multi-Wavelength, Multi-Angle Investigation. J. Appl. Phys. 1998, 83, 3323-3336.

74. Chew, W. C. Waves and Fields in Inhomogeneous Media; Van Nostrand Reinhold: New York, 1990.

75. Kim, J. H.; Ehrman, S. H.; Mulholland, G. W.; Germer, T. A. Polarized Light Scattering by Dielectric and Metallic Spheres on Oxidized Silicon Surfaces. Appl. Opt. 2004, 43, 585-591.

76. Green, M. A.; Keevers, M. J. Optical-Properties of Intrinsic Silicon at 300K. Prog. Photovoltaics 1995, 3, 189-192. 